\title{
¿Cómo y por qué nos formamos los docentes en el uso de tecnología?
}

\author{
Marina Kriscautzky Laxague
}

\section{Resumen}

En este artículo comparto una reflexión sobre la formación docente en el uso de tecnología, por qué es necesaria, la diferencia entre TIC y TAC y cómo afrontar el diseño de procesos formativos desde una perspectiva constructivista.

Palabras clave: formación docente, TIC, TAC, constructivismo.

\section{HOW AND WHY DO TEACHERS TRAIN TO USE TECHNOLOGY?}

\begin{abstract}
In this article I share a reflection on teacher's training in the use of technology, why it is needed, the difference between ICT and TLK, and how to face the design of training processes from a constructivist perspective.
\end{abstract}

Keywords: teacher training, ICT, TLK constructivism.

Dol: http://doi.org/10.22201/codeic.16076079e.2019.v20n6.a2 


\section{Marina Kriscautzky Laxague}

https://orcid.org/0000-0003-3489-1537 mkriscau@unam.mx

Coordinadora de Tecnologías para la Educación, DGTIC-UNAM. Licenciada en Pedagogía por la Facultad de Filosofía y Letras de la unam. Maestra en Ciencias con especialidad en investigación educativa y Doctora en Ciencias con especialidad en investigación educativa en el área de psicolingüística en el Departamento de Investigaciones Educativas (DIE) del CINVESTAV. Ambas tesis dirigidas por la Dra. Emilia Ferreiro.

Ha trabajado en diversos proyectos de inclusión digital, investigación didáctica, desarrollo de materiales digitales para la enseñanza de la lengua y las matemáticas, y formación de docentes en educación básica, media superior y superior en el uso educativo de las tecnologías. Desde 2011 está a cargo de la Coordinación de Tecnologías para la educación —h@bitat Puma- de la Dirección General de Cómputo y de Tecnologías de Información y Comunicación (DGTIC), UNAM, en donde se dedica a la formación de docentes en el uso de la tecnología como herramienta de apoyo a la enseñanza para los niveles medio-superior y superior, la evaluación de habilidades digitales y el desarrollo de estrategias de enseñanza sobre el uso de la tecnología para el aprendizaje en cada área de conocimiento. 


\section{Introducción}

En toda profesión las transformaciones sociales tienen impacto en la manera de ejercerla. Los avances de la ciencia y de la tecnología abren nuevas posibilidades para la práctica de la medicina, la arquitectura o la investigación antropológica, modificando muchas de sus herramientas de trabajo y de sus aproximaciones teóricas a los eventos que estudian o gestionan.

En la docencia pareciera posible cerrar la puerta a las transformaciones externas, pero no es así. Ciertos cambios sociales ejercen presión para modificar la manera en que practicamos nuestra profesión. Es por esto que desde hace varios años las tecnologías de información y comunicación (Tic) han irrumpido en nuestra realidad, convirtiéndose en una aspiración, una obligación o, en muchos casos, un elemento que debe integrarse a pesar nuestro.

Pero... ¿qué uso debemos dar a estas tecnologías? ¿Deben reemplazar todos los medios materiales y tecnologías anteriores? Usar TIC no es una cuestión de moda. Es una necesidad porque estas herramientas ya son prácticas sociales actuales, que nos permiten comunicarnos, acceder

Figura 1. Tecnologías de Información y Comunicación (TIC) en el proceso de tratamiento de la información. a la información y realizar múltiples tipos de intercambios con otras personas. ¿Tiene caso integrarlas a la docencia? Considero que sí, porque son parte de las herramientas culturales que se utilizan en nuestros días y que nuestros estudiantes necesitan aprender a utilizar.

Los estudiantes son hábiles para manejar

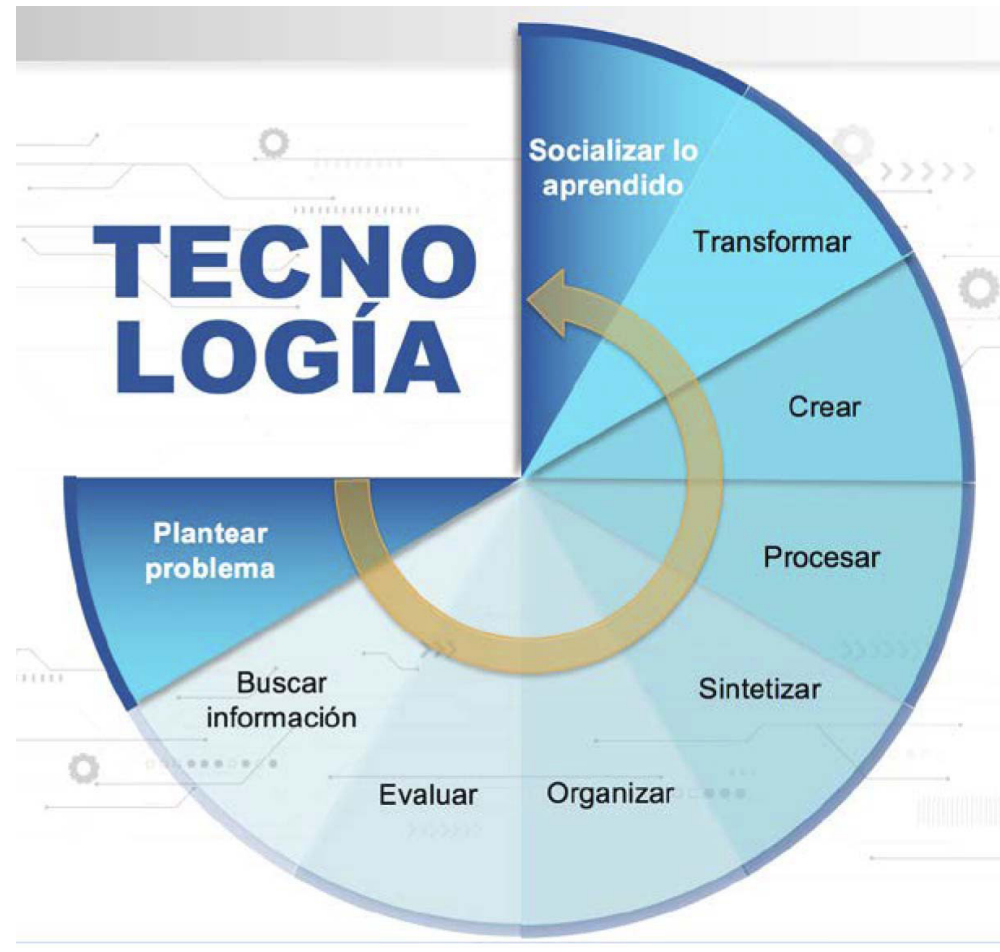
los dispositivos tecnológicos, pero tienen mucho que aprender en relación con el proceso de tratamiento de la información para aprender (Kriscautzky y Ferreiro, 2018; Dirección General de Cómputo y de Tecnologías de Información y Comunicación —DGTIC-, 2018; International Society for Technology in Education —ISTE-, 2016). Cuando estudiamos utilizamos las tic con muchos propósitos. Los principales se pueden observar en la figura 1.

Cuando buscamos información no podemos quedarnos con lo primero que encontramos. Tenemos que evaluarla para decidir si es útil y confiable. Una vez que seleccionamos la información tenemos que organizarla. Para apropiarnos del contenido debemos sintetizar, esquematizar o representar la información obtenida. Seguramente tendremos que procesar la información, es decir, analizarla para poder crear nueva información, algo que podamos comunicar. 
Figura 2. Tecnologías para el Aprendizaje y el Conocimiento (TAC).
En ese proceso transformamos la información en conocimiento. Finalmente, comunicamos lo aprendido a otros.

Para que esto suceda se necesitan profesores capaces de comprender para qué sirven las TIC y de diseñar actividades de aprendizaje donde los estudiantes puedan desarrollar las habilidades digitales que requieren para mejorar su desempeño académico. Este es el eje transversal que nos toca a todos los docentes, independientemente de la asignatura que impartamos. Es así porque formamos personas de manera integral, no pequeños receptáculos de información específica. No pueden desarrollarse esas habilidades en una asignatura en particular, sino a través del uso cotidiano en todas las materias.

Además de este uso transversal de la tecnología, existe otro que los profesores también tenemos que aprender y que está relacionado con las tecnologías para el aprendizaje y el conocimiento (TAC). Las TAC son aquellas tecnologías que nos permiten obtener nuevos modos de representación de los fenómenos (geometría dinámica, imágenes, videos), nuevas formas de acción (procesador textos, simuladores), de experimentación (laboratorios de química o biología) o de exploración (Google Maps).

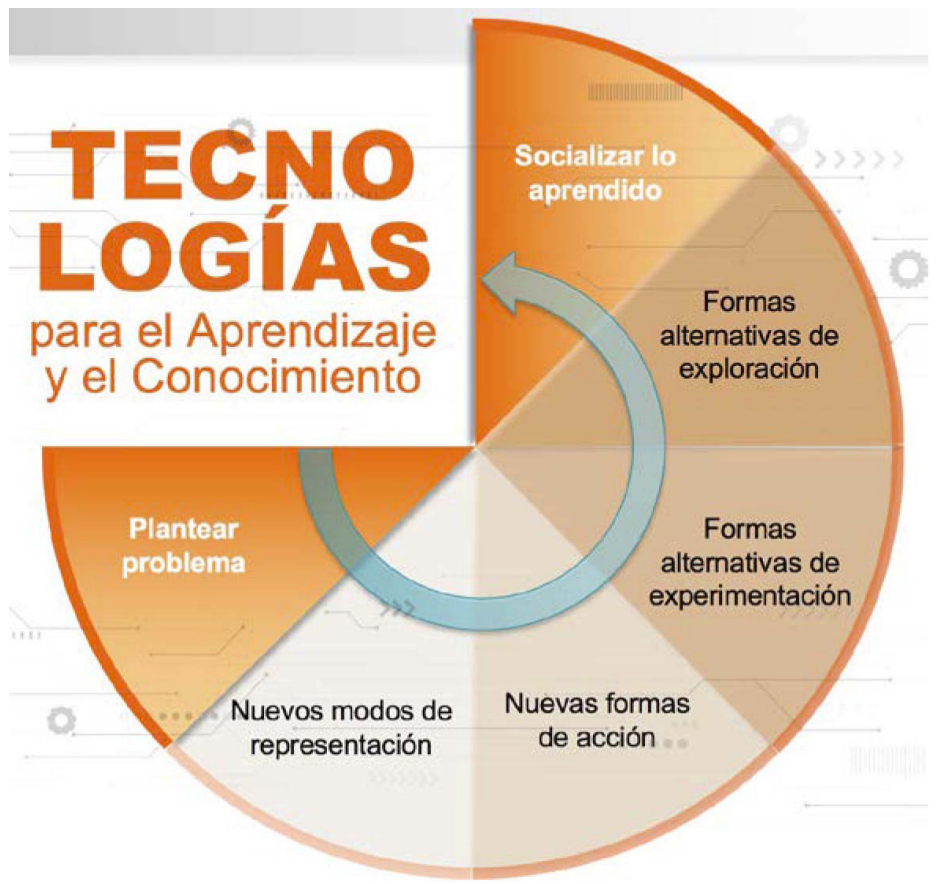

Las TAC están relacionadas con aprendizajes conceptuales o procedimentales específicos, por tanto, no son herramientas transversales sino que están vinculadas a disciplinas particulares. Además, tienen como característica que nos permiten hacer cosas que no podríamos hacer sin esa tecnología o al menos no de forma tan sencilla. Por ejemplo, si en una clase proponemos este problema: ¿el teorema de Pitágoras se cumple con cualquier triángulo o debe ser un triángulo rectángulo?; para resolverlo los estudiantes deberán probar 
por ensayo y error hasta formular una posible respuesta. ¿Pueden hacerlo sin tecnología? Sí, seguramente, pero es muy laborioso y en el camino deberán realizar muchos cálculos donde pueden fallary llegar a conclusiones equivocadas. Si lo hacemos con un software de geometría dinámica (como Geogebra), donde se pueden modificar los ángulos del triángulo y automáticamente se dibujan los cuadrados correspondientes a los tres lados, podrán darse cuenta de que el teorema no vale para cualquier triángulo. La computadora nos ofrece una forma de visualizar los resultados sin preocuparnos por los errores de cálculo que podríamos cometer realizándolos a mano, y de esta forma concentrarnos en elaborar hipótesis y formular respuestas.

Otro ejemplo: el procesador de texto es TAC cuando estamos en la materia Lengua y trabajamos sobre la escritura de un tipo de texto porque permite focalizar su revisión cuando queremos que los estudiantes reflexionen sobre la puntuación o sobre el uso de conectores lógicos. También cuando nos enfocamos en el formato específico de cada tipo textual. Podría hacerse esto sin tecnología, cierto. Lo hicimos por mucho tiempo. Pero al tener esta herramienta podemos introducir estas reflexiones antes con niños pequeños, y con mucha mayor profundidad con jóvenes. Además, existe una ventaja excepcional: corregir, reescribir y formatear no requiere rehacer todo el texto (como cuando lo hacíamos escribiendo a mano o en máquina de escribir). Se puede intervenir el texto en el lugar seleccionado.

Es así como la profesión docente se ve transformada por los avances tecnológicos. Las TAC son los instrumentos particulares de nuestra profesión y es por eso que tenemos que formarnos para utilizarlas cuando es pertinente, para estar al tanto de nuevas herramientas y experiencias exitosas de otros colegas.

\section{Tecnología y transformación de la docencia}

La formación de profesores en el uso de tecnología tiene características particulares ya que no se trata de formar en la enseñanza de una disciplina, como es el caso de la formación en didáctica de las Matemáticas, Historia o Lengua. Se trata de que los profesores se apropien de la tecnología como herramienta para enseñar otras disciplinas. Podría pensarse que es suficiente con enseñarles a manejar determinadas herramientas tecnológicas, es decir, ofrecer una formación técnica, instrumental, dejando que cada uno decida luego cómo hacer que esas herramientas tengan sentido en su práctica docente. También es muy frecuente escuchar afirmaciones como "las Tic transforman la manera de enseñar", "usando tıc dejamos de ser maestros tradicionales", "usar tecnología permite innovar", etcétera. En suma, se le adjudica a la herramienta la transformación de la práctica docente.

Sin embargo, otorgar ese papel a la tecnología trae consecuencias importantes porque lleva a pensar que con dotar de dispositivos e infraestructura estamos "modernizando" la educación. En realidad, el uso que hacemos de la 
Figura 3. Modelo didáctico basado en la transmisión de información. Fuente: needpix.com.
Figuras 4 y $\mathbf{5}$. Modelo didáctico basado en la resolución de problemas y la colaboración. Escuela Nacional Preparatoria plantel No. 6. tecnología no es neutro. La forma en que la empleamos refleja nuestra concepción del aprendizaje. Las herramientas pueden ser las mismas, la diferencia está en cómo las utilizamos: se puede usar TIC y TAC pero continuar con un enfoque de la enseñanza basado en la transmisión de información (ver

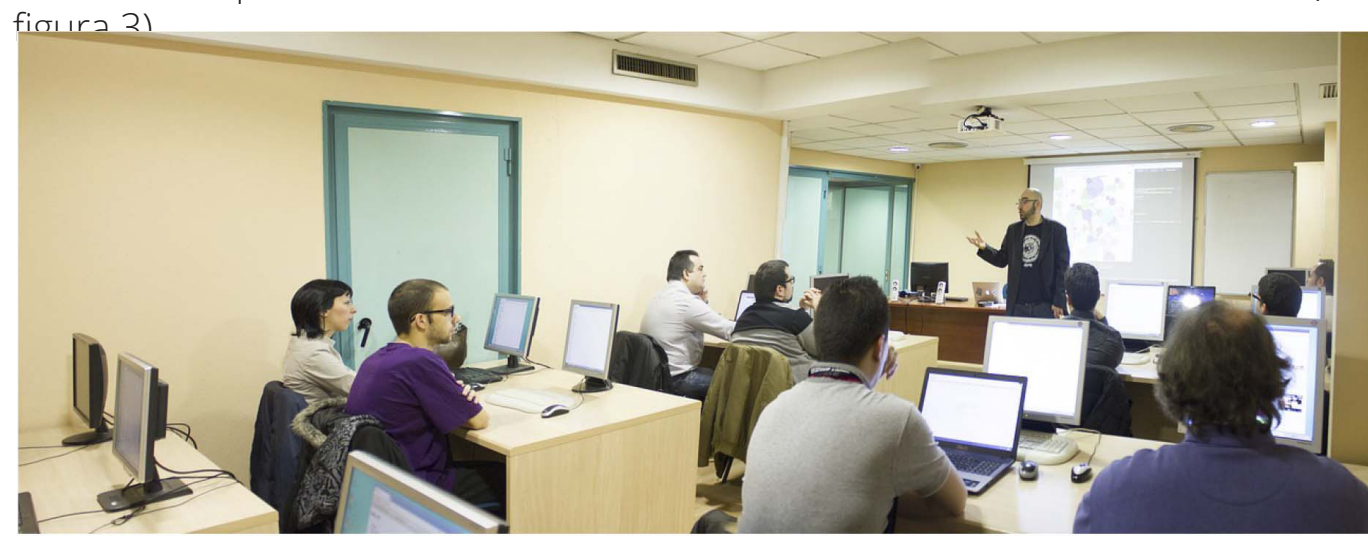

La transformación, en realidad, se relaciona con los profesores, cuando ponen las herramientas en manos de los alumnos para que estos resuelvan problemas relacionados con la disciplina (ver figuras 4 y 5).
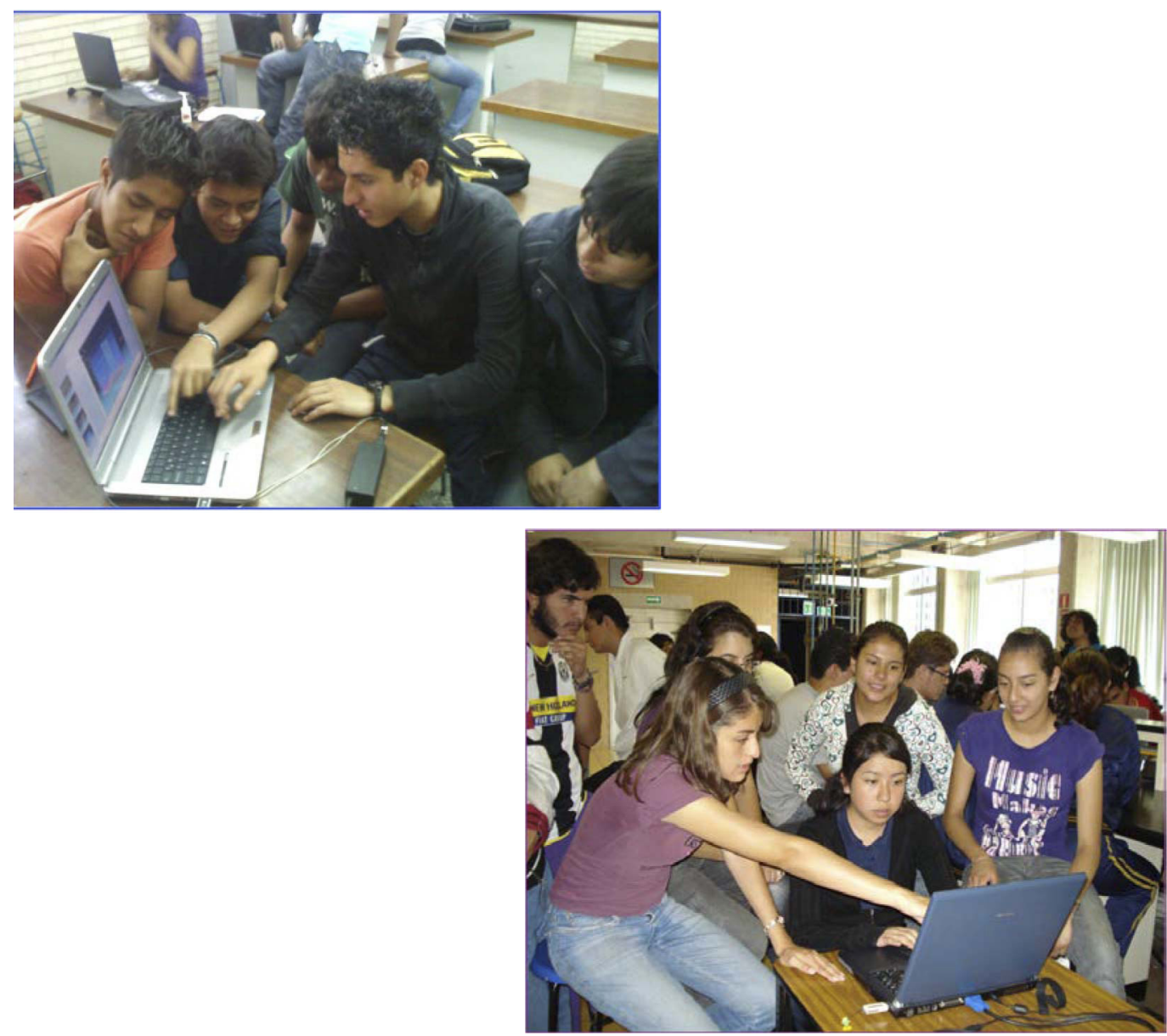
Para que esto suceda, en muchos casos se requiere de un proceso formativo de los docentes enfocado en la reflexión sobre cómo enseñar para utilizar tecnología de la manera más productiva para el aprendizaje. La formación puramente técnica o instrumental no es suficiente.

A partir del enfoque constructivista contamos con trabajos de investigación en formación de profesores que nos permiten plantear referentes para la acción cuando se trata de formar en el uso educativo de la tecnología. Desde esta perspectiva teórica la formación docente se considera como "un proceso continuo y complejo, que no puede resolverse únicamente mediante el aprendizaje de teorías pedagógicas o psicológicas en abstracto o por el entrenamiento de competencias puntuales descontextuadas" (Díaz-Barriga Arceo, 2002: 7).

Ahora bien, vamos a desglosar esta afirmación para aterrizarla en principios más concretos. Comencemos con la idea de que el desarrollo profesional docente es una actividad social y, como tal, debe concebirse como un proceso donde las interacciones son fundamentales para la construcción del conocimiento, de la misma forma que se concibe el aprendizaje de los estudiantes como una construcción social (Bell y Gilbert, 1996; Díaz-Barriga Arceo, 2002).

Aunque el proceso formativo es social, para que un profesor decida incorporarse a un programa de formación es necesario que haya identificado un problema en relación con su práctica docente, ya sea en relación con el aprendizaje de los estudiantes, con su posibilidad de implementar nuevas maneras de enseñar o con su realización profesional (Bell y Gilbert, 1996). Esto es fundamental: nadie inicia un proceso de formación-transformación si no siente la necesidad de hacerlo.

En un proceso formativo es esencial que los profesores tengan la posibilidad de discutir con otros colegas lo que significa ser docente y construir o reconstruir el sentido de la profesión. Sin embargo, aun cuando los profesores se involucran en la formación para resolver problemas de su práctica y aprecian comprender aspectos que no han logrado manejar, también necesitan sentir que su práctica general no es un problema. Se requiere que el formador ayude al valorar a los profesores como docentes competentes en desarrollo, más que como profesores que han fracasado (Bell y Gilbert, 1996).

En esto coincide Lerner y destaca que no se puede pedir a los profesores que abandonen sus prácticas habituales echando por tierra todo lo que saben hacer, sino que es más productivo dar espacio para que vayan incorporando algunas transformaciones en un proceso gradual de construcción de nuevas formas de enseñar:

Esta actitud facilitó mucho más las transformaciones que una excesiva crítica y una compulsividad por el cambio que muy frecuentemente hace caer al docente en una práctica anómica: abandona su anterior manera de enseñar (muchas veces no porque esté muy convencido, sino porque está muy criticado), pero no logra remplazarla por otra práctica organizada y coherente (Lerner, 2001: 189). 
Efectivamente, para integrar nuevas formas de enseñar se necesita construir un nuevo marco de referencia y no hacerlo simplemente porque nos presionan para ello. Sin fundamentos claros las prácticas docentes se convierten en algo de "sentido común" que no tiene sustento y son fácilmente intercambiables por otras, como recetas o tips que "dan resultado".

Aunque esté claro que se requiere construir un nuevo marco de referencia, sabemos que integrar a la práctica nuevas formas de acción es un proceso complejo, porque nuestras creencias y experiencias previas son fuertes y difíciles de transformar. Pero hay algunos caminos probados como efectivos: en primer lugar, es importante que los profesores en formación experimenten situaciones como estudiantes, y que éstas sean muy semejantes a las que ellos generarán en sus propios alumnos. Es decir, ponerlos en "los zapatos" de los estudiantes, resolviendo problemas que hagan activar sus propias representaciones conceptuales de la disciplina. Cuando un docente está en formación se convierte en estudiante; si el proceso formativo promueve la reflexión sobre cómo se enseña y cómo las creencias y preconcepciones intervienen en la práctica, el docente tiene oportunidad de redefinir lo que entiende por ser docente, lo que se concibe por enseñary aprender, y cómo se comprende el objeto de enseñanza (su disciplina). (Liljedahl, 2009; Díaz-Barriga Arceo, 2002; Lerner, 2001)

En este aspecto coincide plenamente Lerner cuando a partir de la experiencia de formación de profesores en el campo de la lectura y la escritura señala:

asumir una concepción constructivista de la enseñanza y el aprendizaje supone centrar la capacitación de los docentes en situaciones que representen un desafío para ellos y les permitan reelaborar el conocimiento, que favorezcan la cooperación entre pares y [...] que permitan explicitar los supuestos implícitos en las posiciones que se adoptan sobre la enseñanza y el aprendizaje (Lerner, 2001, pág. 75).

¿Cómo proponer este tipo de situaciones? Lerner las define como situaciones de doble conceptualización:

[...] aquellas que persiguen un doble objetivo: lograr, por una parte, que los maestros construyan conocimientos sobre un objeto de enseñanza y, por otra parte, que elaboren conocimientos referidos a las condiciones didácticas necesarias para que sus alumnos puedan apropiarse de ese objeto (Lerner, 2001, pág. 173).

Asimismo, los autores que hasta aquí hemos retomado también dan gran importancia al análisis de prácticas de aula para la formación de profesores. Esto es, tomar como material de estudio las experiencias reales de enseñanza, ya sea propias o de docentes expertos. (Gellert, 2009; Díaz-Barriga Arceo, 2002). Lerner agrega una característica fundamental al analizar situaciones de aula

que pueden caracterizarse como "buenas", porque son estas situaciones las que permiten explicitar el modelo didáctico con el que se trabaja, [...] porque los interrogantes que el capacitador plantea sobre su desarrollo conducen a elaborar conclusiones positivas acerca de la naturaleza del contenido que se está enseñando y aprendiendo en esa clase así como sobre las intervenciones del docente y los efectos producidos por cada una de ellas (Lerner, 2001, pág. 179). 
Finalmente, destaca la importancia del estudio bibliográfico en los procesos de formación como un aspecto que permite a los docentes comprender y fundamentar las prácticas de aula y volverse cada vez más independiente en su proceso de construcción de conocimientos:

El trabajo sobre la bibliografía cumple un papel importante, porque es a través de esas lecturas como los maestros pueden reflexionar acerca de las propuestas didácticas sobre las cuales están trabajando, así como sobre sus fundamentos y porque conocer a los diferentes autores les permitirá manejarse con autonomía para avanzar en su propia formación más tarde, cuando ya no estén involucrados en un curso de capacitación (Lerner, 2001, pág. 76).

En suma, concebir la formación docente desde una perspectiva constructivista requiere, ante todo, ser coherente con dicha perspectiva concibiendo al profesor en formación como un sujeto que aprende construyendo conocimientos a través de la resolución de problemas y de la interacción con otros. Para formar profesores no basta con ofrecer contenido, hay que diseñar situaciones que activen sus concepciones previas y los enfrenten a la necesidad de construir nuevos conocimientos, propiciar la reflexión sobre la práctica y ofrecer lecturas teóricas que les permitan encontrar explicaciones y fundamentos a las propuestas didácticas que están construyendo (Díaz-Barriga Arceo, 2002; Solbes y González, 2016).

\section{De lo ideal a lo posible}

Todos quisiéramos contar con las mejores condiciones para formarnos como profesores: formación inicial con prácticasmonitoreadas pormentores; formación en servicio con acompañamiento constante en los primeros años; seminarios de discusión; actualización permanente; espacios para la investigación educativa, etcétera (Díaz-Barriga Arceo, 2002).

Sin embargo, las condiciones reales de las instituciones públicas como nuestra Universidad imponen muchas restricciones relacionadas con el financiamiento, tiempos, espacios, reconocimientos y condiciones para la formación docente, por mencionar algunas. Entonces, se vuelve imperativo identificar qué es posible dentro de cada contexto y cómo, a partir de ello, sacar el mayor provecho para que la formación tenga impacto en lo que cada profesor puede hacer para transformar su práctica. En este contexto, quisiera rescatar los elementos que considero fundamentales y que deberían estar presentes en mayor o menor medida en las propuestas formativas.

En primer lugar, la formación en el uso de tecnología no puede, o mejor dicho no debe, estar disociada de los procesos de formación docente en los diferentes campos disciplinares. Usar tecnología conlleva, explícita o implícitamente, una postura acerca del aprendizaje.

Durante la formación es importante promover la interacción entre los docentes para formar comunidades de práctica y reflexión sobre la práctica. Esa 
reflexión debe realizarse a partir de la teoría, encontrando en ella las respuestas a los problemas didácticos que se quieren resolver (Solbes y González, 2016; Díaz-Barriga Arceo, 2002; Lerner, 2001).

Finalmente, es necesario construir con los profesores referentes para la práctica a través de las situaciones de doble conceptualización, de manera que ante una situación nueva en el aula cuenten con experiencias provistas por la formación para producir respuestas (y no recurrir a las que tuvieron como alumnos porque no necesariamente fueron consistentes con el enfoque constructivista).

Por ejemplo, producir un texto argumentativo con fuentes digitales es una situación en la que los profesores aprenden a utilizar fuentes en internet, a escribir en un procesador con un formato específico y a emplear herramientas colaborativas para la revisión centrada en la argumentación. Luego, en un segundo momento, pueden reflexionar acerca de las condiciones didácticas que hicieron posibles esos aprendizajes, las intervenciones del formador, la forma de interactuar con sus pares y el papel que jugaron las herramientas tecnológicas. Esto les dará una nueva experiencia que será referente a la hora de intentar realizar esta actividad con sus estudiantes.

\section{Conclusiones}

Ser profesor es un desafío porque la docencia es una profesión que requiere múltiples conocimientos y habilidades. La integración de tecnologías es inherente a la práctica docente. El uso que hagamos de esas tecnologías pone de manifiesto nuestra concepción de aprendizaje, nuestro dominio de la disciplina que enseñamos, y de su didáctica, sobre las cuales debemos estar actualizados de manera permanente.

Las acciones de formación docente en el uso de tecnología deben mantener coherencia entre la teoría y la práctica, porque en las actividades que se desarrollan para la formación se pueden construir nuevos referentes para la acción en el aula. Y, sobre todo, para no reproducir aquello que decimos que queremos transformar.

\section{Referencias}

* Kriscautzky Laxague, M y Ferreiro, E. (2018, enero-marzo). Evaluar la confiabilidad de la información en Internet: cómo enfrentan el reto los nuevos lectores de 9 a 12 años. Perfiles educativos, xL(159), 16-34.

Bell, B., y Gilbert, J. (1996). Teacher Development: A Model From Science Education. London: The Falmer Press.

* Dirección General de Cómputo y de Tecnologías de Información y Comunicación (DGTIC). (2018). Informe de resultados del TICómetro, diagnóstico de habilidades en el uso de tic para estudiantes de primer ingreso al bachillerato de la unAm. México: unAm. 
* Díaz-Barriga Arceo, F. (2002). Aportaciones de las perspectivas constructivista y reflexiva en la formación docente en el bachillerato. Perfiles Educativos, 24 (97-98), 6-25.

* Gellert, U. et al. (2009). 1.1.3 Practising Mathematics Teacher Education: Expanding The Realm of Possibilities. En Ruhama Even y Deborah Loewenberg Ball (Eds.), The Professional Education and Development of Teachers of Mathematics The 15th IcmI Study, (35-55). EuA: Springer.

* International Society for Technology in Education (ISTE). (2016). ISTE Standards for Students. A Practical Guide for Learning with Technology. EUA: ISTE.

* Lerner, D. (2001). Leer y escribir en la escuela: lo real, lo posible y lo necesario. México: SEP- Fondo de Cultura Económica.

* Liljedahl, P. et al. (2009). Chapter 1.1.2 Components of Mathematics Teacher Training. En Ruhama Even y Deborah Loewenberg Ball (Eds.), The Professional Education and Development of Teachers of Mathematics The 15th Icm Study, (35-55). EUA: Springer.

* Organisation for Economic Co-operation and Development (oECD). (2016). PISA 2018. Draft analytical frameworks. May 2016. Recuperado de: https://www.oecd. org/pisa/data/PISA-2018-draft-frameworks.pdf.

* Solbes,J.,y González, E. (2016, enero-junio). Aportes a la formación del profesorado constructivista: resultados en dos países. Praxis y Saber, 7(13), 63-88.

\section{Cómo citar este artículo}

* Kriscautzky Laxague, Marina (2019). ¿Cómo y por qué nos formamos los docentes en el uso de tecnología? Revista Digital Universitaria (RDU). Vol. 20, núm. 6 noviembrediciembre. Dol: http://doi.org/10.22201/codeic.16076079e.2019.v20n6.a2.

Recepción: 23/07/2019. Aprobación: 20/09/2019 